REVISTA CIENCIAS BIOMÉ DICAS

ARTÍCULOS DE REVISIÓN

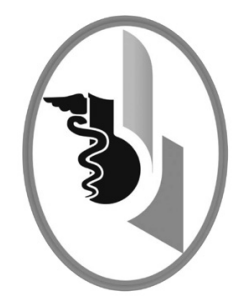

\title{
RENDIMIENTO DEL HE4 EN EL DIAGNÓSTICO, PRONÓSTICO Y PREDICCIÓN DE RECIDIVA EN CÁNCER DE ENDOMETRIO
}

\author{
HE4 PERFORMANCE IN THE DIAGNOSIS, PROGNOSIS AND \\ PREDICTION OF RELAPSE IN ENDOMETRIAL CANCER
}

\author{
Puentes-Puentes Luis Orlando ${ }^{1}$ \\ Amaya-Pino Juan Pablo² \\ Correspondencia: orlando-puentes@hotmail.com \\ Recibido para evaluación: marzo - 25 - 2015. Aceptado para publicación: octubre - 15 - 2016
}

\section{RESUMEN}

Introducción: el cáncer de endometrio es un cáncer ginecológico frecuente. La biopsia de endometrio es la prueba de oro para el diagnóstico. Ningún biomarcador ha mostrado relevancia clínica hasta el momento. El HE4 parece ser de alta sensibilidad y especificidad, incluso en los estadios tempranos.

Objetivos: analizar la evidencia médica publicada hasta la fecha sobre el potencial valor del HE4 como prueba diagnóstica, al igual que su utilidad en lo concerniente al pronóstico y recaída en las pacientes con cáncer de endometrio.

Materiales y métodos: se realizó revisión narrativa de la literatura en las bases de datos Pubmed-Medline y EMBASE desde 1990 a 2016. La búsqueda se estructuró utilizando términos MESH.

Resultados: los resultados de sensibilidad varían según los diferentes puntos de cortes de HE4 entre $41 \%$ y $94 \%$, especificidad entre $36 \%$ y $100 \%$. El punto de corte que mejor rendimiento mostró fue $70 \mathrm{pmol} / \mathrm{L}$, con sensibilidad de $59.4 \%$ y especificidad de $100 \%$. En pronóstico, los estudios muestran que el HE4 puede ayudar en la predicción del riesgo de invasión miometrial, para la adecuada estatificación, así como definición del compromiso extrauterino y ganglionar para determinar de una manera más acertada la necesidad de linfadenectomía.

Conclusión: resultados iniciales sugieren que el HE4 es un biomarcador que puede ser útil en el diagnóstico de las mujeres con cáncer de endometrio, además puede identificar aquellas con alto riesgo de recurrencia y puede ser utilizado en el seguimiento y detección de esta. Rev.cienc.biomed. 2016;7(2):274-284.

PALABRAS CLAVE

Neoplasia endometrial; Endometrio; Biopsia.

1 Docente asociado. Servicio de Ginecología Oncológica. Fundación Universitaria de Ciencias de la Salud (FUCS). Hospital de San José. Bogotá, Colombia.

2 Estudiante de especialidad. Ginecología Oncológica. Fundación Universitaria de Ciencias de la Salud (FUCS). Hospital de San José. Bogotá, Colombia. 


\section{SUMMARY}

Introduction: endometrial cancer is a common gynecologic cancer. Endometrial biopsy is the gold standard for diagnosis. No biomarker has shown clinical relevance until now. The HE4 seems to be a biomarker with high sensitivity and specificity, even in the early stages.

Objective: this review aims to analyze the medical evidence published to date about the potential value of HE4 as a diagnostic test, as well its usefulness regarding the prognosis and relapse in patients with endometrial cáncer.

Material and methods: a narrative review of the literature was carried out by means of PubMed-MEDLINE and EMBASE databases from 1990 to 2016. The search was structured using MeSH terms.

Results: 18 studies were identified. The sensitivity results vary according to the different cut-off points of HE4 between $41 \%$ and $94 \%$; specificity between $36 \%$ and $100 \%$. The best cut-off performance was $70 \mathrm{pmol} / \mathrm{L}$ with a sensitivity of $59.4 \%$ and specificity of $100 \%$. In prognosis, studies showed that HE4 can help to predict the risk of myometrial invasion, adequate stratification and extrauterine and nodal involvement to determine in a more accurate way the necessity or not necessity of lymphadenectomy. Conclusion: the initial results suggest that HE4 serves as a biomarker in the diagnosis of women with endometrial cancer. Also it is able to identify those women with high recurrence risk; it can be used to detect and monitor the aforementioned conditions.

Rev.cienc.biomed. 2016;7(2):274-284.

\section{KEYWORDS}

Endometrial neoplasm; Endometrium; Biopsy.

\section{INTRODUCCIÓN}

El cáncer de endometrio es un cáncer ginecológico frecuente, precedido por cáncer de mama, colon, cérvix y pulmón (1). En Estados Unidos fue estimado en 54.870 nuevos casos en el año 2015 con 10.170 muertes (2). La incidencia del cáncer de endometrio a nivel mundial alcanza hasta 8.2 por 100.000 mujeres/año y una mortalidad de 1.8 por 100.000 mujeres/año (3). En Colombia la incidencia es de 3.6 por 100.000 mujeres/año y una mortalidad de 1 por 100.000 mujeres/año (4).

Los síntomas más comunes son sangrado uterino anormal y flujo vaginal. Los pacientes con estadio avanzado tienen síntomas similares a los observados con cáncer de ovario avanzado, tales como dolor pélvico, distensión abdominal, saciedad temprana o el cambio en función intestinal, o de la vejiga. La evaluación inicial incluye examen físico y biopsia endometrial (5).

La exposición prolongada a estrógenos sin oposición se asocia con la mayoría de los cánceres de endometrio tipo I. La terapia de reemplazo hormonal de solo estrógenos aumenta el riesgo de cáncer de endometrio de dos a veinte veces, y este aumento se correlaciona con la duración del uso (6).
La exposición a estrógenos endógenos sin oposición, como ocurre en el síndrome de ovario poliquístico, tumores productores de estrógenos y produce la conversión periférica excesiva de los andrógenos a estrona en el tejido adiposo, también se asocia con un mayor riesgo de desarrollar hiperplasia endometrial y cáncer (6).

El tamoxifeno, un modulador selectivo del receptor estrogénico, actúa como un estrógeno antagonista en los tejidos de mama y como agonista en el endometrio. El uso del tamoxifeno se asocia con un aumento de seis veces en la incidencia de cáncer de endometrio (7).

La obesidad tiene un profundo impacto en el incremento de la incidencia de cáncer de endometrio. Esto puede explicarse por el aumento de la producción endógena de estrógenos a través de la aromatización en los tejidos adiposos. Además, antes de la menopausia, las mujeres obesas pueden tener mayor riesgo de diabetes mellitus y anovulación crónica, lo cual se asocia con un mayor riesgo de cáncer endometrial (8).

La hipertensión se ha asociado con un mayor riesgo de cáncer endometrial, pero comorbilidades médicas tales como la diabetes y la obesidad se comportan como 
una variable de confusión en esta asociación (9). Características reproductivas como la infertilidad, la nuliparidad, la menarquia temprana y la menopausia tardía también se han descrito como factores de riesgo para cáncer de endometrio (10).

Durante muchos años la ecografía transvaginal se ha utilizado para la evaluación de mujeres con sangrado postmenopáusico. El objetivo de la ecografía es excluir carcinoma de endometrio, dado que con un endometrio delgado menor a cinco $\mathrm{mm}$ la posibilidad de este es menor al $1 \%$ y así, se puede evitar la toma de biopsias innecesarias. En muchos países las guías de manejo aconsejan la medición del espesor endometrial por ecografía transvaginal como un primer paso en la evaluación de la mujer con sangrado postmenopáusico.

La mayoría de estas guías se basan en recomendaciones dadas por el meta-análisis Smith-Bindman et al., publicado en JAMA 1998, en el que un punto de corte de $5 \mathrm{~mm}$ tiene sensibilidad del $96 \%$ para la detección de cáncer de endometrio. Tal combinación de sensibilidad y especificidad reduciría la probabilidad pre-test de $10 \%$ para el cáncer de endometrio a una probabilidad post-test de $1 \%$ (11). Si el punto de corte se reduce a $3 \mathrm{~mm}$, la sensibilidad mejora al $98 \%$ pero la especificidad disminuye al 35\% (11).

La biopsia guiada por histeroscopia es el estándar de oro para detección de cáncer endometrial, sin embargo, la biopsia de endometrio con cánula de aspiración de pipelle tiene una sensibilidad de $99 \%$ para cáncer de endometrio, por lo cual también puede ser utilizada $(12,13)$.

La identificación de los ganglios linfáticos metastásicos por tomografía o resonancia magnética nuclear, que se basa en la medición del tamaño de los ganglios, mostró para diámetros mayores a $10 \mathrm{~mm}$ una sensibilidad que oscila entre $27 \%$ y $66 \%$ y una especificidad entre $73 \%$ y $99 \%$ (14). La sensibilidad, especificidad, valor predictivo positivo, valor predictivo negativo de PET/TC en la detección de metástasis en los ganglios linfáticos son $50 \%, 90.9 \%$, $66.7 \%$ y $83.3 \%$ respectivamente, con una exactitud del $80 \%$ (15).
El pronóstico depende del estadio de la enfermedad, aproximadamente el $70 \%$ de los casos son diagnosticados en estadios tempranos y el incremento en la mortalidad y disminución en el período libre de recurrencia están directamente relacionados con un incremento en los estadios avanzados y aparición de tipos histológicos no favorables como seroso papilar y células claras (16). El tipo y el grado histológico, tamaño tumoral, invasión linfovascular, compromiso cervical y ganglios linfáticos son variables pronósticas que se asocian con recurrencia (16).

Existen varios estudios de evaluación de marcadores tumorales séricos individuales en cáncer endometrial como M-CSF, CA19.9, CEA, CA-15.3 y CA-72.4, en los que se encontraron resultados elevados en el $30 \%$ de los pacientes (16).

En la actualidad, la detección de la enfermedad recurrente es a través del seguimiento de los síntomas clínicos y el uso de técnicas de imagen, que normalmente detectan la enfermedad en estadios avanzados. Se ha demostrado que los niveles de CA-125 en suero se correlacionaron con la presencia de la enfermedad extrauterina, metástasis peritoneales y linfáticas (16). Sin embargo, el CA-125 para estadio I tiene una sensibilidad muy baja, solo del $20 \%$ y especificidad del $95 \%$, y para los pacientes con enfermedad avanzada sensibilidad de $32 \%$ y especificidad del 95\% (17).

Además, el CA-125 es poco específico porque puede encontrarse elevado en muchas patologías como el cáncer de mama, endometrio, ovario, pulmón y otras condiciones como quistes benignos, cirrosis, endometriosis, embarazo. En resumen, el CA125 es un biomarcador con baja sensibilidad y especificidad para el cáncer de endometrio y su utilidad radica en la monitorización de la respuesta clínica (18). Por otro lado, el HE4 ha demostrado ser un biomarcador con mejor sensibilidad y especificidad, incluso en los estadios tempranos de pacientes con cáncer de endometrio (18). El objetivo fue reunir la evidencia que se ha publicado hasta la fecha sobre el valor de HE4 en el diagnóstico, pronóstico y recurrencia de las pacientes con cáncer de endometrio. 


\section{MATERIALES Y MÉTODOS}

Se realizó revisión narrativa de la literatura desde 1990 hasta marzo 2016. La búsqueda se estructuró utilizando los términos MESH Endometrial Neoplasm, (OR Neoplasm, Endometrial OR Neoplasms, Endometrial OR Endometrial Carcinoma OR Carcinoma, Endometrial OR Carcinomas, Endometrial OR Endometrial Carcinomas OR Endometrial Cáncer) y human epididymal secretory protein E4 (OR EDDM4 protein, human OR HE4 protein, human OR ESP-H4 protein, human OR WAP5 protein, human OR WAP four-disulfide core domain 2 protein, human OR human epididymis-specific protein E4) en las bases de datos PUBMED-MEDLINE, EMBASE. Se utilizó como filtro "humans" y como operador booleano el término AND. Se incluyeron estudios publicados en formato de texto completo y se dividieron los estudios según su finalidad, diagnóstico, pronóstico y predicción de recaída.

\section{RESULTADOS}

En las dos bases de datos se encontraron 74 artículos, siendo 18 seleccionados para la revisión. En Pubmed-Medline se identificaron 43, siendo descartados 17 por referirse a patologías diferentes al cáncer de ovario, 12 por no ser de contenido clínico y dos por ser revisiones de la literatura. Fueron seleccionados 12 artículos. En EMBASE se encontraron 31 artículos diferentes a los ya seleccionados, 14 fueron descartados por tratar temas diferentes al cáncer de endometrio, 10 por carecer de contenido clínico, uno era revisión de la literatura y se consideraron apropiados 6 artículos.

\section{DISCUSIÓN}

La proteína 4 humana secretora del epidídimo HE4, también conocido como WFDC2, es un nuevo marcador conocido en el cáncer de ovario; fue descubierto por Kirchhoff en 1991. Desde el 2008 viene siendo valorado como biomarcador en el cáncer de endometrio en estadios tempranos. El HE4 se clonó por primera vez como una de cuatro proteínas expresadas en el epidídimo humano. La secuencia de aminoácidos sugiere que el HE4 pertenece a la familia WAP. El gen del HE4 se encuentra en el cromosoma 20q13.12, una región que alberga un grupo de genes para proteínas de dominio $\operatorname{WAP}(18,19)$. Es bien sabido que al menos algunos miembros de esta familia sirven como inhibidores de la proteasa de péptidos endógenos, pero la función de HE4 no se ha aclarado completamente. El HE4 presenta cuatro variantes: V1, V2, V3, V4 $(19,20)$.

Utilidad diagnóstica. Sobre el valor que tiene el HE4 como herramienta diagnóstica en el cáncer de endometrio se encontraron varios artículos que informan sobre su sensibilidad y especificidad (1,16-18,21-24). Tabla No 1.

Omer publicó en el 2013 un estudio que evaluó la eficacia predictiva de HE4 y el suero amiloide $A(S-A A)$ junto con los otros

\begin{tabular}{|c|c|c|c|c|}
\hline \multicolumn{5}{|c|}{$\begin{array}{l}\text { TABLA No } 1 . \\
\text { RENDIMIENTO DEL HE4 EN EL DIAGNÓSTICO DE CÁNCER DE ENDOMETRIO }\end{array}$} \\
\hline Estudio & $\begin{array}{c}\mathrm{N}^{\circ} \text { de } \\
\text { pacientes }\end{array}$ & $\begin{array}{l}\text { Punto de corte } \\
\text { HE4 pmol/L }\end{array}$ & Sensibilidad (\%) & Especificidad (\%) \\
\hline Omer (2013) & 158 & 59.7 & 75.0 & 65.5 \\
\hline Moore (2008) & 327 & - & 45.5 & 95.0 \\
\hline Zanotti (2012) & 318 & 51.0 & 78.8 & 84.8 \\
\hline Zhang (2012) & 330 & - & 41.1 & 95.0 \\
\hline Angioli (2013) & 204 & 70.0 & 59.4 & 100.0 \\
\hline Bignotti (2011) & 214 & - & 67.0 & 95.0 \\
\hline Kemik (2016) & 100 & $\begin{array}{l}36.3 \\
38.9\end{array}$ & $\begin{array}{l}94.0 \\
90.0\end{array}$ & $\begin{array}{l}36.0 \\
42.0\end{array}$ \\
\hline
\end{tabular}


marcadores tumorales en pacientes con cáncer de endometrio. Los niveles de HE4 y S-AA fueron mayores en los pacientes con cáncer que en los controles. El mejor punto de corte para HE4 fue 59.7 pmol/L. Concluyó que la medición preoperatoria de HE4 en suero podía ayudar en la detección temprana de cáncer endometrial (16).

Moore realizó un estudio en pacientes diagnosticadas con cáncer de endometrio y un grupo control a la cuales se les realizó de forma preoperatoria medición en sangre de los niveles de CA-125, CA-72.4, HE4 y SMRP. Se construyeron curvas ROC para encontrar la mejor sensibilidad y especificidad para cada uno de estos biomarcadores y combinación entre ellos. Sus hallazgos sugieren que el HE4 puede ser útil en la evaluación y detección de enfermedad recurrente pre-clínica, la respuesta a la terapia, como indicador de pronóstico y en la evaluación preoperatoria de las pacientes con cáncer de endometrio (17). Además anotaron que el HE4 fue significativamente mejor en comparación con CA-125 en la diferenciación del cáncer de endometrio y patología benigna (17).

En un estudio realizado por Zanotti et al. se encontró que el HE4 presentó mejor rendimiento diagnóstico que el CA-125 para el diagnóstico de mujeres con cáncer de endometrio. Con el punto de corte de $51 \mathrm{pmol} / \mathrm{L}$ la especificidad de HE4 para discriminar cáncer de endometrio de patología benigna fue del $84.8 \%$ y la sensibilidad fue de $78.8 \%$ (18).

Zhang realizó un trabajo en el que comparó los niveles en suero de HE4 y CA-125 de mujeres con cáncer de endometrio, enfermedad benigna del útero y mujeres sanas. Se pudo determinar que los niveles de HE4 y CA-125 en el suero del grupo de carcinoma endometrial fueron significativamente mayores que los del grupo de enfermedad benigna de útero o grupo de mujeres sanas $(P<0.05)$. Las tasas de positividad de HE4 y CA-125 fueron del $31 \%$ y $12 \%$ en el estadio I-II respectivamente, mientras que las tasas de positividad en estadios III-IV fueron del $63 \%$ y $47 \%$, con lo cual el autor concluyó que la sensibilidad y especificidad del HE4 en el diagnóstico precoz son mejores que la del
CA-125 y a su vez, las tasas de positividad se relacionaron con el estado clínico (21).

Por otra parte, Angioli et al., incluyeron pacientes con cáncer de endometrio y un grupo control con patología benigna, en dicho grupo se realizó determinación en sangre de HE4 y CA-125 y se estableció que el punto de corte de $70 \mathrm{pmol} / \mathrm{L}$ es el que presenta la mejor sensibilidad y especificidad con $59.4 \%$ y $100 \%$ respectivamente, valor predictivo positivo $100 \%$ y VPN $71.5 \%$. Esto sugiere que la determinación del HE4 en suero puede ayudar a determinar adecuadamente el estado de la lesión, antes de la cirugía definitiva (22).

Bignotti et al. publicaron un trabajo cuyo objetivo fue determinar los niveles séricos de HE4 en pacientes con cáncer de endometrio frente a otras sin patología endometrial, con el fin de correlacionar los niveles del mismo con las características clínico-patológicas y variables pronosticas. Se obtuvo como resultado que los niveles de HE4 fueron significativamente más altos en las pacientes con cáncer de endometrio que en los controles (23).

Finalmente, Kemik en el 2016, publicó su trabajo, cuyo objetivo fue determinar la eficacia de YKL-40, He4 y DKK 3 en el diagnóstico temprano de pacientes con cáncer de endometrio y en la estimación preoperatoria de los parámetros de pronóstico tales como el estadio, el grado y la extensión de la enfermedad. Los niveles preoperatorios de CA125, HE4 y YKL-40 fueron significativamente mayores en el grupo de cáncer de endometrio $(p<0.001)(24)$.

Utilidad pronóstica. Se identificaron 14 artículos que determinaron dicha utilidad según los niveles séricos de HE4 y los valoraron según invasión miometrial, compromiso ganglionar o estadio clínico (16, 18, 22-33). Tabla N².

Aunque la mayoría de las pacientes con cáncer de endometrio tienen pronóstico favorable, existe una pequeña proporción de tumores que muestran un comportamiento agresivo como los grados histológico tipo tres, los histológicos no endometrioide, 


\begin{tabular}{|c|c|c|c|c|c|c|c|c|c|c|c|c|c|c|c|}
\hline \multirow{12}{*}{ 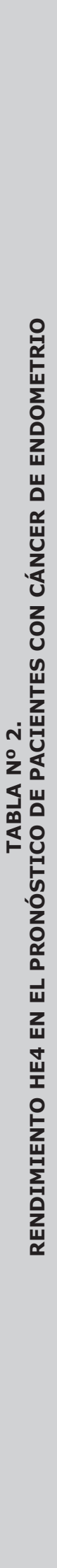 } & 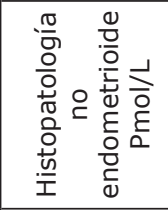 & i & i & $\begin{array}{l}\infty \\
\dot{\varphi} \\
\text { in }\end{array}$ & i & i & i & i & $\begin{array}{l}\circ \\
\stackrel{1}{ }\end{array}$ & $\begin{array}{l}+\dot{0} \\
\text { ó } \\
\text { ஸे } \\
\text { ñ }\end{array}$ & $\begin{array}{l}0 \\
\dot{m} \\
\infty \\
m\end{array}$ & i & $\begin{array}{l}\infty \\
\dot{\infty} \\
\infty\end{array}$ & i & i \\
\hline & 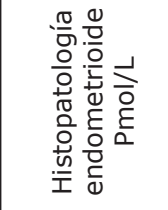 & i & $\begin{array}{l}\infty \\
\stackrel{\infty}{\infty}\end{array}$ & $\begin{array}{l}\hat{0} \\
\stackrel{m}{m} \\
-1\end{array}$ & i & i & i & i & $\begin{array}{l}0 \\
\infty \\
\infty\end{array}$ & $\begin{array}{l}0 \\
\dot{\sigma}\end{array}$ & $\stackrel{N}{\emptyset}$ & i & $\hat{n}$ & i & i \\
\hline & 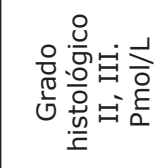 & $\begin{array}{l}\stackrel{0}{0} \\
\dot{b} \\
\stackrel{-}{-1}\end{array}$ & $\begin{array}{l}+ \\
\substack{+\infty}\end{array}$ & $i$ & i & 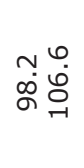 & i & $\begin{array}{l}\text { N. } \\
\text { Oे }\end{array}$ & $\begin{array}{l}\circ \\
\stackrel{\infty}{\infty}\end{array}$ & $\begin{array}{l}\dot{v} \\
m \\
o \\
\infty \\
\infty \\
\dot{\infty}\end{array}$ & i & 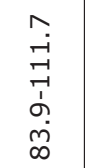 & $\hat{\text { i }}$ & 宅 & $\begin{array}{l}\vec{I} \\
\overrightarrow{1} \\
\stackrel{1}{N}\end{array}$ \\
\hline & 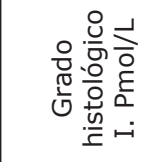 & $\begin{array}{l}0 \\
\infty \\
\infty \\
\infty\end{array}$ & $\begin{array}{l}\stackrel{\infty}{\oplus} \\
\stackrel{\oplus}{0}\end{array}$ & i & i & $\stackrel{\stackrel{゙}{n}}{\stackrel{n}{n}}$ & i & $\begin{array}{l}\infty \\
\dot{0} \\
\dot{\theta}\end{array}$ & $\begin{array}{l}\stackrel{0}{0} \\
\dot{0}\end{array}$ & $\begin{array}{l}\text { Ln } \\
\text { in }\end{array}$ & i & $\hat{\infty}$ & $\stackrel{\vec{n}}{\stackrel{n}{n}}$ & 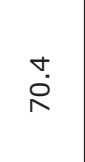 & $\begin{array}{l}\dot{0} \\
\dot{0}\end{array}$ \\
\hline & 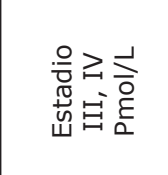 & $\stackrel{\circ}{\stackrel{M}{N}}$ & i & i & 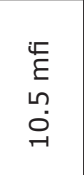 & i & i & $\begin{array}{l}0 \\
\infty \\
\infty \\
+1\end{array}$ & $\begin{array}{l}\stackrel{ }{\mathrm{i}} \\
\underset{7}{-}\end{array}$ & 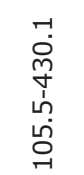 & $i$ & 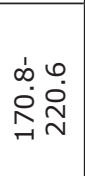 & $\begin{array}{l}\stackrel{0}{\dot{P}} \\
\stackrel{m}{-}\end{array}$ & $\begin{array}{l}0 \\
\stackrel{0}{0} \\
\text { - }\end{array}$ & 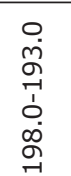 \\
\hline & 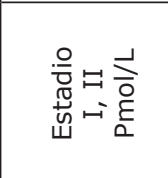 & $\begin{array}{l}\circ \\
\text { ळ }\end{array}$ & $\begin{array}{l}0 \\
\text { ம் } \\
0\end{array}$ & i & 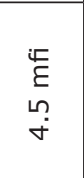 & $\begin{array}{l}\infty \\
\infty \\
\infty \\
+1 \\
+1 \\
0 \\
i \\
N\end{array}$ & ' & $\stackrel{N}{N}$ & $\begin{array}{l}\stackrel{\circ}{+} \\
\stackrel{+}{n}\end{array}$ & $\begin{array}{l}0 \\
\dot{+} \\
\dot{1} \\
\dot{y} \\
\dot{v}\end{array}$ & i & $\begin{array}{l}\stackrel{N}{ } \\
\dot{m} \\
\stackrel{-}{-} \\
\infty \\
\dot{0} \\
\dot{0}\end{array}$ & $\hat{\mathfrak{N}}$ & 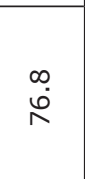 & $\begin{array}{l}0 \\
\infty \\
0 \\
ت 1 \\
1 \\
0 \\
\dot{m} \\
0\end{array}$ \\
\hline & 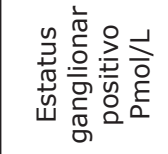 & ' & 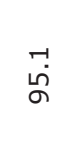 & i & 1 & i & $\begin{array}{l}0 \\
\stackrel{g}{q}\end{array}$ & $i$ & $\begin{array}{l}0 \\
\text { á }\end{array}$ & 足 & $\begin{array}{l}\overrightarrow{1} \\
\stackrel{-}{I} \\
-1\end{array}$ & 1 & i & 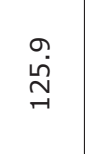 & ! \\
\hline & 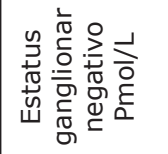 & ' & $\begin{array}{l}\text { no } \\
\stackrel{n}{R}\end{array}$ & i & i & i & in & ! & $\begin{array}{l}\stackrel{\circ}{\Gamma} \\
\stackrel{+}{N}\end{array}$ & $\stackrel{m}{n}$ & $\begin{array}{l}\hat{0} \\
\stackrel{\rho}{\circ}\end{array}$ & i & i & $\begin{array}{l}\infty \\
\stackrel{\infty}{\wedge}\end{array}$ & : \\
\hline & 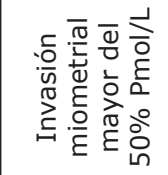 & $\stackrel{\circ}{\stackrel{M}{\sim}}$ & $\begin{array}{l}\circ \\
\text { m̄ }\end{array}$ & $\begin{array}{l}\hat{0} \\
\stackrel{\infty}{\infty} \\
\stackrel{-}{-1}\end{array}$ & $\begin{array}{l}\stackrel{L}{E} \\
\varepsilon \\
\stackrel{L}{0} \\
\stackrel{\emptyset}{-1}\end{array}$ & i & $\begin{array}{l}\text { Ln } \\
\stackrel{0}{0}\end{array}$ & 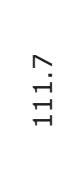 & $\begin{array}{l}0 \\
\infty \\
\infty\end{array}$ & $\begin{array}{l}\qquad 0 \\
\infty \\
\infty \\
\infty\end{array}$ & $\begin{array}{l}\text { Na } \\
\stackrel{\infty}{\sim} \\
\sim\end{array}$ & i & $\begin{array}{l}m \\
\stackrel{m}{\omega} \\
\rightarrow\end{array}$ & $\begin{array}{l}0 \\
0 \\
0 \\
-1\end{array}$ & $\underset{\stackrel{n}{m}}{\stackrel{n}{G}}$ \\
\hline & 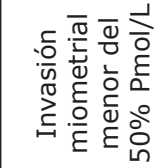 & $\begin{array}{l}\circ \\
\text { ळ }\end{array}$ & \begin{tabular}{l}
\multirow{2}{*}{} \\
$\stackrel{0}{0}$
\end{tabular} & 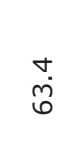 & $\begin{array}{l}\stackrel{\mathfrak{L}}{\varepsilon} \\
\stackrel{m}{\sigma}\end{array}$ & i & $\hat{o}$ & $\begin{array}{c}m \\
\infty \\
0 \\
0\end{array}$ & $\begin{array}{l}0 \\
\dot{\theta} \\
\dot{b}\end{array}$ & $\hat{\text { ஸે }}$ & में & i & $\begin{array}{l}0 \\
0 \\
0 \\
0\end{array}$ & 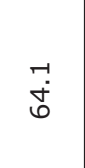 & $\begin{array}{l}\circ \\
\dot{R}\end{array}$ \\
\hline & 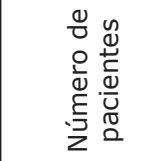 & $\stackrel{\substack{n \\
\sim}}{n}$ & $\underset{\sigma}{m}$ & 굼 & $\stackrel{\text { n }}{\wedge}$ & $\stackrel{\infty}{\wedge}$ & 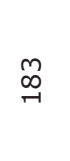 & $\stackrel{\infty}{\circ}$ & 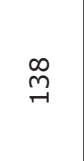 & 放 & 우 & $\stackrel{N}{N}$ & $\stackrel{m}{m}$ & ஜ & 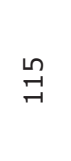 \\
\hline & 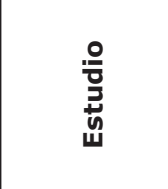 & 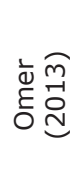 & 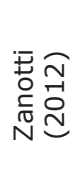 & $\begin{array}{l}\overline{\bar{m}} \\
\overline{\bar{\sigma}} \overline{0} \\
\overline{0}\end{array}$ & 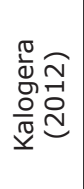 & 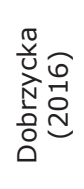 & 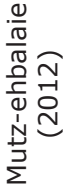 & 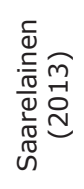 & 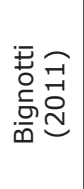 & 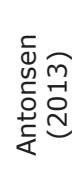 & 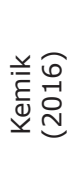 & 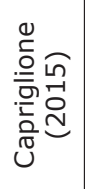 & 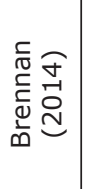 & 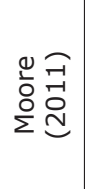 & 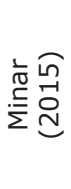 \\
\hline
\end{tabular}


los que tienen compromiso ganglionar e invasión miometrial profunda. Por lo tanto, se deben valorar diferentes biomarcadores tanto de diagnóstico como de pronóstico que permitan una adecuada estratificación de los pacientes, para mejorar estrategias de tratamiento (16). Omer et al. establecieron que los niveles séricos de HE4 se relacionaron con el grado histológico y la estatificación clínica del tumor. La tamización preoperatoria con este marcador puede proporcionar información importante acerca del pronóstico del paciente (16).

Zanotti et al., en el 2012, indicaron que el HE4 parece estar asociado con un patrón más agresivo y podría ser de utilidad para la identificación de pacientes con cáncer de endometrio de alto riesgo. El HE4 se correlacionó con la disminución de la supervivencia global y la supervivencia libre de enfermedad (18). Anglioli reportó que HE4 suero puede determinar la estratificación preliminar del riesgo antes de la cirugía definitiva y establecer al HE4 como una nueva herramienta para evaluación preoperatoria y postoperatoria de vigilancia para pacientes con cáncer de endometrio (22).

Un tema controversial en el manejo del cáncer de endometrio es la necesidad de linfadenectomía. Tres ensayos controlados aleatorizados investigaron el papel de la linfadenectomía en el cáncer de endometrio. Un primer estudio realizado por Benedetti et al. y el segundo estudio del grupo ASTEC, publicado en 2009 por Kitchene, concluyeron que la linfadenectomía pélvica no puede ser recomendada como procedimiento de rutina para fines terapéuticos fuera de los ensayos clínicos $(34,35)$. Por otro lado, el estudio SEPAL, publicado en el 2010, estableció que combinando la linfadenectomía pélvica y paraaórtica se mejora la sobrevida global de pacientes con carcinoma de endometrio de riesgo intermedio y alto (36).

Kalogera et al. publicaron en el 2011 un estudio en el que se evaluó el HE4 como marcador para la enfermedad de alto riesgo en pacientes con cáncer endometrial. Incluyó 75 pacientes tratados quirúrgicamente con cáncer endometrial y se midieron los niveles de HE4 preoperatorio para compararlos con un grupo control. El nivel de HE4 en cáncer de endometrio fue significativamente más elevado en pacientes con invasión miometrial mayor del 50\% (estadio IB), que en aquellas con invasión miometrial menor del $50 \%$ (estadio IA) y con mayor tamaño tumoral, pareciendo ser más sensible que el CA-125. Estos hallazgos sugieren que el HE4 podría servir como un predictor preoperatorio sobre la necesidad de realizar linfadenectomía (25).

Otro estudio que sugiere la utilidad del HE4 ante la necesidad de linfadenectomía en cáncer de endometrio, realizado por Dobrzycka, cuyo objetivo fue establecer si los niveles séricos preoperatorios de HE4 y CA-125 podrían ser buenos predictores de la necesidad de linfadenectomía en la etapa inicial de adenocarcinoma endometrioide del útero. Se midieron en 78 pacientes posmenopáusicas tratadas quirúrgicamente, observándose que HE4 de 78 pmol/L fue el mejor punto de corte para identificar candidatas a requerir linfadenectomía con sensibilidad de $86.6 \%$ y la especificidad del $67.2 \%$. El nivel de corte de CA-125 que mostró mejores índices pronósticos fue 26U/ $\mathrm{ml}$, con sensibilidad de $66.6 \%$ y especificidad del $61.2 \%$. Los resultados indican que en etapa temprana de cáncer endometrial tipo endometrioide, el HE4 puede servir como herramienta preoperatoria y puede ayudar a identificar en posmenopáusicas cuáles pueden requerir linfadenectomía (26).

Mutz-Dehbalaie et al. realizaron un estudio cuyo objetivo fue evaluar el valor pronóstico de HE4 previo al tratamiento en pacientes con cáncer de endometrio en comparación con el CA-125. Se analizaron HE4 y CA-125 en 183 muestras en suero de pacientes con cáncer de endometrio, se determinó el valor pronóstico de HE4, CA-125 y la combinación de ambos marcadores; un punto de corte de HE4 de $81 \mathrm{pmol} / \mathrm{L}$ fue usado para discriminar como bajos o altos los niveles de HE4. Para el CA-125 se estableció un punto de corte de $35 \mathrm{U} / \mathrm{ml}$. Se encontró que tanto el HE4 y CA-125 fueron capaces de discriminar entre buen y mal pronóstico de supervivencia global y libre de enfermedad. En el análisis multivariado el HE4 mostró una clara ventaja en la información pronóstica en comparación 
con CA-125. En la sobrevida libre de progresión ninguno de estos marcadores por sí solos, ni sus combinaciones tuvieron valor pronóstico (27).

En el 2013 se publicó el trabajo realizado por Saarelainen, cuyo objetivo fue evaluar el desempeño del HE4 en la predicción de la presencia de metástasis en carcinoma de endometrio, con un punto de corte de $70 \mathrm{pmol} / \mathrm{L}$ y $35 \mathrm{U} / \mathrm{ml}$ de HE4 y CA-125, respectivamente. Las concentraciones séricas de HE4 y CA-125 fueron mayores en el grupo con metástasis que en el grupo sin metástasis. La combinación de HE4 y CA-125 fue el único factor predictivo para la presencia de metástasis con OR: 21.5; la sensibilidad y especificidad de la combinación de los marcadores para predecir la presencia de metástasis fueron $71.4 \%$ y $89.5 \%$, respectivamente. Una combinación de HE4 y CA-125 preoperatoria parece ser un adecuado predictor de la enfermedad metastásica en pacientes con cáncer de endometrio (28).

Bignotti estableció que los niveles altos de HE4 eran significativamente asociados con peores características clínicas de las pacientes con cáncer de endometrio. Los niveles altos de HE4 se correlacionaron con una menor supervivencia global y menor tiempo de supervivencia libre de progresión (23). Antonsen, en el estudio ENDOMET, quiso establecer si el HE4 y el CA-125 se correlacionan con factores pronósticos conocidos para alto riesgo de cáncer de endometrio. Los niveles de los biomarcadores se correlacionaron con las características patológicas de especímenes de histerectomía. Se encontró que tanto HE4 y CA-125 se correlacionaban de forma positiva con el grado histológico, metástasis de ganglios linfáticos ( $p<0.0001)$, invasión del miometrio $(p<0.0001)$ y el compromiso cervical ( $p<0.0001)$. Además del estadio, el índice combinado puede ayudar a los ginecólogos en la predicción del riesgo de invasión miometrial profunda, el compromiso cervical y las metástasis a ganglios linfáticos (29).

Kemik estableció que los niveles séricos de $\mathrm{HE}-4$ fueron significativamente mayores en los estadios avanzados ( $p$ <0.004) y en pacientes con invasión linfovascular. Adicionalmente, HE4 se correlacionó significativamente con factores pronósticos y puede tener éxito en la determinación temprana de cáncer de endometrio, y en la detección de subconjuntos de alto riesgo antes de la cirugía (24).

En ese mismo sentido, Capriglione publicó un trabajo en el que determinó la correlación entre los niveles preoperatorios de HE4 en pacientes con cáncer de endometrio y el punto de corte ideal para la predicción del estadio, el tipo histológico endometrioide vs. no endometrioide y el grado histológico. Se consideró un punto de corte HE4 de $70 \mathrm{pmol} / \mathrm{L}$. Se encontró que los niveles más altos de HE4 se correlacionaban con grado histológico $3(p<0.05)$ y con estadio avanzado lo que sugiere un papel potencial de HE4 en la predicción del estadio en cáncer de endometrio (30).

Brennan estimó la utilidad HE4 en suero como un biomarcador para fenotipos de alto riesgo en pacientes con cáncer. Midió los niveles en suero de HE4 y CA-125 perioperatorio en cáncer de endometrio identificado a partir del Estudio de cáncer de endometrio nacional de Australia (ANECS). La mediana de los niveles de CA-125 y HE4 fueron mayores en estadio III y IV $(p=0.001)$ y con invasión del miometrio mayor de $50 \%$ ( $p<0.001)$. El HE4 fue un mejor predictor de invasión del miometrio mayor del $50 \%$ que el CA-125, especialmente en pacientes con tumores de bajo grado endometrioide. Se demostró que la elevación de la HE4 fue un predictor independiente de supervivencia libre de recurrencia (HR: 2.40; $p=0.014$ ), con lo que demostró la utilidad de HE4 como un biomarcador de pronóstico en cáncer endometrial.

Se observó que existe utilidad del HE4 preoperatorio para identificar pacientes de alto riesgo con cáncer endometrial tipo endometrioide de bajo grado, que podrían beneficiarse de linfadenectomía. Estos hallazgos son similares a los reportados en el estudio de Moore, el cual concluyó que el HE4 puede ser un marcador útil en el proceso preoperatorio para la toma de 
decisiones clínicas, especialmente para determinar la necesidad de una disección de ganglios linfáticos en las mujeres con cáncer endometrial tipo endometrioide (31-33).

Utilidad para riesgo de recurrencia. Al respecto se encontraron dos estudios $(37,38)$. El objetivo del trabajo de Angioli fue evaluar en la División de Oncología Ginecológica del Campus Biomédico de la Universidad de Roma el papel de HE4 al momento del diagnóstico primario, en comparación con CA-125 como indicador de recurrencia para cáncer de endometrio. Fue un análisis retrospectivo de 252 pacientes tratadas con cáncer de endometrio, de ellas 37 experimentaron recurrencia. Los niveles de HE4 y CA-125 fueron analizados al momento del diagnóstico primario, durante el seguimiento y después de la confirmación de recurrencia. Se observó una diferencia estadísticamente significativa entre los valores de HE4 al momento del diagnóstico primario y en la recurrencia, respectivamente, comparando los pacientes recurrentes y no recurrentes $(p<0.05)$, mientras que los valores de CA-125 no resultaron estadísticamente significativos $(p<0.08)$. Los niveles de HE4 en el diagnóstico primario se correlacionaron con un mayor riesgo de recurrencia de cáncer de endometrio, en particular en los casos de histopatología endometrioide, y pueden ayudar a reconocer a las pacientes que pueden necesitar un mayor seguimiento intensivo (37).

A su vez Brennan evaluó la HE4 en suero como un biomarcador para detectar enfermedad recurrente durante el seguimiento de pacientes con cáncer de endometrio.
De igual manera, realizó un análisis retrospectivo de 98 pacientes, de las cuales 26 desarrollaron la enfermedad recurrente. La mediana de seguimiento fue de cinco años. Los niveles de HE4 disminuyeron después del tratamiento inicial $(p<0.001)$ y aumentaron de nuevo en la recidiva $(p=0.002)$. En histología endometrioide, y el HE4 en suero medido durante el seguimiento era un mejor indicador de recurrencia que el CA125. El nivel HE4 de $70 \mathrm{pmol} / \mathrm{L}$ se asoció con sensibilidad del $84 \%$ y especificidad del $74 \%$ en la evaluación de cáncer de endometrio tipo endometrioide recurrente (38).

\section{CONCLUSIÓN}

El HE4 se viene considerando como el biomarcador más sensible y específico para cáncer de endometrio en el momento, siendo superior al CA-125 en el cribado. En particular, este nuevo marcador parece tener un buen desempeño en el diagnóstico, incluso en las primeras etapas. Se encontró que el mejor punto de corte del HE4 en el diagnóstico de cáncer de endometrio es $70 \mathrm{pmol} / \mathrm{L}$, con baja sensibilidad y buena especificidad en todos los estadios. Se ha señalado su capacidad para discriminar invasión miometrial, compromiso extrauterino y ganglionar, mejorando la indicación para linfadenectomía y contribuyendo a reducir la morbilidad relacionada con su realización. Tendría utilidad en el seguimiento y detección la recurrencia.

CONFLICTO DE INTERESES: ninguno que declarar.

FINANCIACIÓN: recursos propios de los autores.

\section{REFERENCIAS BIBLIOGRÁFICAS}

1. Bie Y, Zhang Z. Diagnostic value of serum HE4 in endometrial cancer: a meta-analysis. World J Surg Oncol. 2014;12:169.

2. NCCN. Clinical practice gidelines in oncology. Uterine Neoplasms version 2.20162016 .

3. World Health Organization, International Agency for Research on Cancer. Globocan 2012. Estimated cancer incidence, mortality and prevalence worldwide in 2012 [Internet]. Geneve: The Organization; 2015 [cited 2015/11/05]. Available from: http://globocan.iarc.fr/Default. aspx.

4. Pardo-Ramos C, Cendales-Duarte R. Incidencia, mortalidad y prevalencia de cáncer en Colombia, 2007-2011. Instituto Nacional de Cancerología. 2015.

5. Burke WM, Orr J, Leitao M, Salom E, Gehrig P, Olawaiye AB, et al. Endometrial cancer: a review and current management strategies: part I. Gynecol Oncol. 2014;134(2):385-92. 
6. Pike MC, Peters RK, Cozen W, Probst-Hensch NM, Felix JC, Wan PC, et al. Estrogen-progestin replacement therapy and endometrial cancer. J Natl Cancer Inst. 1997;89(15):1110-6.

7. Fisher B, Costantino JP, Redmond CK, Fisher ER, Wickerham DL, Cronin WM. Endometrial cancer in tamoxifen-treated breast cancer patients: findings from the National Surgical Adjuvant Breast and Bowel Project (NSABP) B-14. J Natl Cancer Inst. 1994;86(7):527-37.

8. Soliman PT, Wu D, Tortolero-Luna G, Schmeler KM, Slomovitz BM, Bray MS, et al. Association between adiponectin, insulin resistance, and endometrial cancer. Cancer. 2006;106(11):2376-81.

9. Soler M, Chatenoud L, Negri E, Parazzini F, Franceschi S, la Vecchia C. Hypertension and hormone-related neoplasms in women. Hypertension. 1999;34(2):320-5.

10. McPherson CP, Sellers TA, Potter JD, Bostick RM, Folsom AR. Reproductive factors and risk of endometrial cancer. The Iowa Women's Health Study. Am J Epidemiol. $1996 ; 143(12): 1195-202$.

11. Timmermans A, Opmeer BC, Khan KS, Bachmann LM, Epstein E, Clark TJ, et al. Endometrial thickness measurement for detecting endometrial cancer in women with postmenopausal bleeding: a systematic review and meta-analysis. Obstet Gynecol. 2010;116(1):160-7.

12. Epstein E, Ramirez A, Skoog L, Valentin L. Dilatation and curettage fails to detect most focal lesions in the uterine cavity in women with postmenopausal bleeding. Acta Obstet Gynecol Scand. 2001;80(12):1131-6.

13. Dijkhuizen FP, Mol BW, Brölmann HA, Heintz AP. The accuracy of endometrial sampling in the diagnosis of patients with endometrial carcinoma and hyperplasia: a meta-analysis. Cancer. $2000 ; 89(8): 1765-72$.

14. Connor JP, Andrews JI, Anderson B, Buller RE. Computed tomography in endometrial carcinoma. Obstet Gynecol. 2000;95(5):692-96.

15. Kitajima K, Murakami K, Yamasaki E, Kaji Y, Sugimura K. Accuracy of integrated FDG-PET/ contrast-enhanced CT in detecting pelvic and paraaortic lymph node metastasis in patients with uterine cancer. Eur Radiol. 2009;19(6):1529-36.

16. Omer B, Genc S, Takmaz O, Dirican A, Kusku-Kiraz Z, Berkman S, et al. The diagnostic role of human epididymis protein 4 and serum amyloid-A in early-stage endometrial cancer patients. Tumour Biol. 2013;34(5):2645-50.

17. Moore RG, Brown AK, Miller MC, Badgwell D, Lu Z, Allard WJ, et al. Utility of a novel serum tumor biomarker HE4 in patients with endometrioid adenocarcinoma of the uterus. Gynecol Oncol. 2008;110(2):196-201.

18. Zanotti L, Bignotti E, Calza S, Bandiera E, Ruggeri G, Galli C, et al. Human epididymis protein 4 as a serum marker for diagnosis of endometrial carcinoma and prediction of clinical outcome. Clin Chem Lab Med. 2012;50(12):2189-98.

19. Jiang SW, Chen H, Dowdy S, Fu A, Attewell J, Kalogera E, et al. HE4 transcription- and splice variants-specific expression in endometrial cancer and correlation with patient survival. Int J Mol Sci. 2013;14(11):22655-77.

20. Galgano MT, Hampton GM, Frierson HF. Comprehensive analysis of HE4 expression in normal and malignant human tissues. Mod Pathol. 2006;19(6):847-53.

21. Zhang AM, Zhang P. [Clinical value of combined detection of serum human epididymal secretory protein E4 and $\mathrm{CA}(125)$ in the diagnosis of endometrial carcinoma]. Zhonghua Fu Chan Ke Za Zhi. 2012;47(2):125-8.

22. Angioli R, Plotti F, Capriglione S, Montera R, Damiani P, Ricciardi R, et al. The role of novel biomarker HE4 in endometrial cancer: a case control prospective study. Tumour Biol. 2013;34(1):571-6.

23. Bignotti E, Ragnoli M, Zanotti L, Calza S, Falchetti M, Lonardi S, et al. Diagnostic and prognostic impact of serum HE4 detection in endometrial carcinoma patients. $\mathrm{Br}$ J Cancer. 2011;104(9):1418-25.

24. Kemik P, Saatli B, Yıldırım N, Kemik VD, Deveci B, Terek MC, et al. Diagnostic and prognostic values of preoperative serum levels of YKL-40, HE-4 and DKK-3 in endometrial cancer. Gynecol Oncol. 2016;140(1):64-9.

25. Kalogera E, Scholler N, Powless C, Weaver A, Drapkin R, Li J, et al. Correlation of serum HE4 with tumor size and myometrial invasion in endometrial cancer. Gynecol Oncol. 2012;124(2):270-5.

26. Dobrzycka B, Mackowiak-Matejczyk B, Terlikowska KM, Kinalski M, Terlikowski SJ. Utility of HE4 to identify patients with endometrioid endometrial cancer who may require lymphadenectomy. Adv Med Sci. 2016;61(1):23-7.

27. Mutz-Dehbalaie I, Egle D, Fessler S, Hubalek M, Fiegl H, Marth C, et al. HE4 is an independent prognostic marker in endometrial cancer patients. Gynecol Oncol. 2012;126(2):186-91.

28. Saarelainen SK, Peltonen N, Lehtimäki T, Perheentupa A, Vuento MH, Mäenpää JU. Predictive value of serum human epididymis protein 4 and cancer antigen 125 concentrations in endometrial carcinoma. Am J Obstet Gynecol. 2013;209(2):142.e1-6. 
29. Antonsen SL, Høgdall E, Christensen IJ, Lydolph M, Tabor A, Loft Jakobsen A, et al. HE4 and CA125 levels in the preoperative assessment of endometrial cancer patients: a prospective multicenter study (ENDOMET). Acta Obstet Gynecol Scand. 2013;92(11):1313-22.

30. Capriglione S, Plotti F, Miranda A, Ricciardi R, Scaletta G, Aloisi A, et al. Utility of tumor marker HE4 as prognostic factor in endometrial cancer: a single-center controlled study. Tumour Biol. 2015;36(6):4151-6.

31. Brennan DJ, Hackethal A, Metcalf AM, Coward J, Ferguson K, Oehler MK, et al. Serum HE4 as a prognostic marker in endometrial cancer a population based study. Gynecol Oncol. 2014;132(1):159-65.

32. Moore RG, Miller CM, Brown AK, Robison K, Steinhoff M, Lambert-Messerlian G. Utility of tumor marker HE4 to predict depth of myometrial invasion in endometrioid adenocarcinoma of the uterus. Int J Gynecol Cancer. 2011;21(7):1185-90.

33. Minar L, Klabenesova I, Jandakova E, Zlamal F, Bienertova-Vasku J. Prognostic value of human epididymis protein 4 in endometrial cancer and its utility for surgical staging. J Obstet Gynaecol Res. 2015;41(10):1644-52.

34. Benedetti Panici P, Basile S, Maneschi F, Alberto Lissoni A, Signorelli M, Scambia G, et al. Systematic pelvic lymphadenectomy vs. no lymphadenectomy in early-stage endometrial carcinoma: randomized clinical trial. J Natl Cancer Inst. 2008;100(23):1707-16.

35. Kitchener H, Swart AM, Qian Q, Amos C, Parmar MK, group As. Efficacy of systematic pelvic lymphadenectomy in endometrial cancer (MRC ASTEC trial): a randomised study. Lancet. 2009;373(9658):125-36.

36. Todo $Y$, Kato H, Kaneuchi M, Watari H, Takeda M, Sakuragi N. Survival effect of para-aortic lymphadenectomy in endometrial cancer (SEPAL study): a retrospective cohort analysis. Lancet. 2010;375(9721):1165-72.

37. Angioli R, Capriglione S, Scaletta G, Aloisi A, Miranda A, De Cicco Nardone C, et al. The role of HE4 in endometrial cancer recurrence: how to choose the optimal follow-up program. Tumour Biol. 2016;37(4):4973-78.

38. Brennan DJ, Hackethal A, Mann KP, Mutz-Dehbalaie I, Fiegl H, Marth C, et al. Serum HE4 detects recurrent endometrial cancer in patients undergoing routine clinical surveillance. BMC Cancer. 2015;15:33.

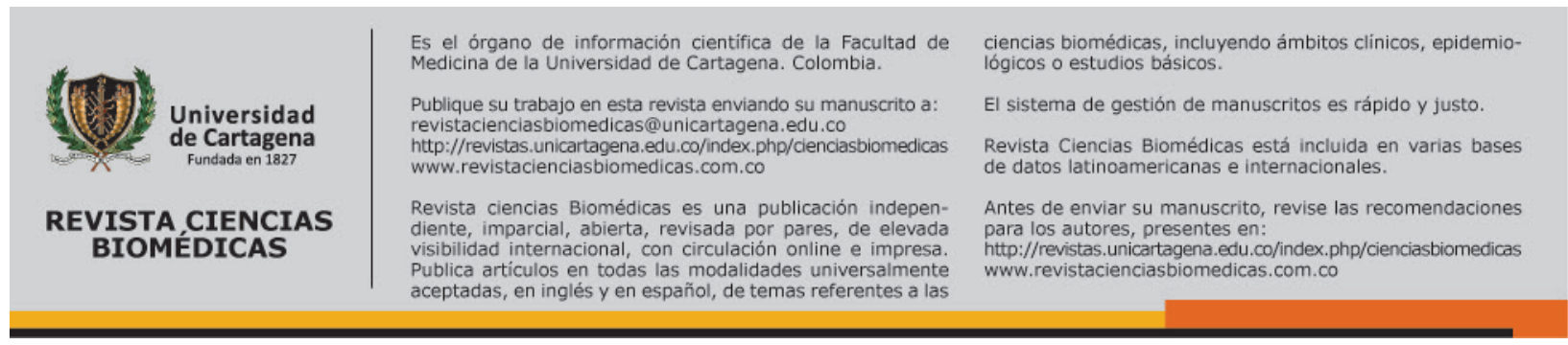

